\title{
A Deep Learning Based Suggested Model to Detect Necrotising Enterocolitis in Abdominal Radiography Images
}

\author{
Jacqueline van Druten \\ School of Architecture, Computing and \\ Engineering, College of \\ Arts, Technology and Innovation, UEL \\ University Way, Dockland Campus \\ London, E16 2RD, UK. \\ u1639256@uel.ac.uk
}

Clara Chong

Neonatal unit, Evelina Children's

Hospital NHS Foundation Trust

Westminster Bridge Rd, Lambeth,

London SE1 7EH, UK.

\author{
Mhd Saeed Sharif \\ School of Architecture, Computing and \\ Engineering, College of \\ Arts, Technology and Innovation, UEL \\ University Way, Dockland Campus \\ London, E16 2RD, UK. \\ s.sharif@uel.ac.uk \\ Hassan Abdalla \\ School of Architecture, Computing and \\ Engineering, College of \\ Arts, Technology and Innovation, UEL \\ University Way, Dockland Campus \\ London, E16 2RD, UK.
}

\author{
Sherwin S Chan \\ Department of Radiology \\ Children's Mercy Hospital \\ BH15 2JB, UK \\ Kansas City, MO, USA, 64108.
}

\begin{abstract}
Despite decades of exploration into necrotising enterocolitis (NEC), we still lack the capacity to accurately diagnose the disease to improve outcomes in its management. Existing diagnostics struggle to delineate NEC from other neonatal intestinal diseases; it is also unable to highlight those likely to deteriorate to needing emergency life-saving surgery before it is too late. The diagnosis of NEC is heavily dependent on interpretation of radiological findings, especially abdominal radiography (AR) and abdominal ultrasound (AUS). Interexpert variability in interpreting AR imaging, and in the case of AUS, performing and interpreting the test, remains an unresolved challenge. With the compounding impact of the shrinking radiology workforce, a novel approach is imperative. Computer assisted detection (CAD) and classification of abnormal pathology in medical imaging is a rapidly evolving field of clinical and biomedical research. This technology is widely used as a preliminary screening tool. This research paper proposes a deep learning-based model to classify $A R$ images in an automated manner, generating class activation maps (CAM) from various imaging features consistent with NEC pathology, as agreed by expert consensus papers (in neonatology and paediatric radiology). It also compares it with conventional machine learning methods. The suggested model aims to produce heatmaps for various imaging features to highlight NEC pathology in AR (or in future AUS). Once the model is trained, validation is done through quantitative measures and visually by the attending radiologist (clinician) reviewing the validity of the colour maps highlighting the pathology of the AR image (future extension to AUS). As the volume of imaging data is increasing year by year, CAD can be a key strategy to assist radiology departments meet service needs. This technology can greatly assist in screening for NEC, improving the detection of NEC and potentially aid in the earlier identification of disease. Furthermore, it can fast track research cost effectively by creating big data through the automatic labeling of imaging data to create big-data for NEC databases.
\end{abstract}

Keywords- Computer assisted detection, Necrotizing enterocolitis, Abdominal radiograph, Abdominal X-ray, CAD,
LBP, SVM, CNN, Machine learning, artificial intelligence, Deep learning, Ensemble Modelling, Class activation map.

\section{INTRODUCTION}

Necrotizing enterocolitis (NEC) is one of the main causes death and disability in preterm newborns. It is estimated that one in a thousand live-born infants develop NEC and one in four diagnosed with NEC deteriorate to require emergency life-saving surgery[1]. For those that require emergency surgery, $46,5 \%$ do not survive. $25 \%$ of those survivors develop life-altering co-morbidities such as short bowel syndrome or impaired neuro-development [2], [3]. Despite significant investment in time and resources, our understanding of the causation of NEC has not progressed enough to improve outcomes [4]-[7]. The lack of progress in outcomes for NEC is, to a large extent, related to a lack of consensus for a case definition and data sets could better inform practice but are difficult to aggregate and are contaminated and as such limited, unreliable and inaccurate in the insights they can offer [5], [8]. Much hope still hinges on biomarker research [8], but for now, we are still without reliable biomarkers to predict or detect NEC early or delineate it from various confounders.

The most recent Bliss reports[9] in the U.K. and similar reports from various global charities[10], highlight the lifelong devastating impact of prematurity and NEC on families and the significant socio-economic burden this condition places on society. Premature infants (24-34 weeks' gestation) will typically stay in the hospital until about 36-44 gestation. NEC exaggerates this cost by lengthening the average length of stay to approximately 20 days longer for medical NEC and about 61 days longer for surgical NEC [11], [12]. NEC can account for nearly a fifth of the yearly neonatal unit expenditure. It is estimated that one in a thousand live-born infants develop NEC, and one in four infants progress to surgical NEC [13]. There is currently no international report 
on the socio-economic burden but a US-based report estimated that $\$ 5$ billion per year is spent on NEC hospitalization, with NEC medical care costs of \$216 666 per survivor and a potential cost saving of $\$ 200000$ per patient could be achieved if progression to surgery is prevented [12].

Long-term neurodevelopment has become a critical area in NEC research, shifting the focus from survival alone to minimising impairment as the primary goal. RECAP study demonstrated that prematurity and LBW were associated with poorer wealth markers as it showed lower rates of tertiary qualifications, increased risk unemployment, and an increased risk of dependence on social welfare in adulthood [10]. NEC that progresses to surgical management compounds this more as larger areas on brain MRI are adversely affected and higher rates of neurodevelopmental impairments (NDI) seen in follow-up neurodevelopmental checks [14]. Surgical NEC significantly increases the odds ratio of cerebral palsy (mean=1,55), visual impairment (mean=2,31), cognitive impairment (mean=1,44) and psychomotor impairment (mean=1,72). Given the above, the early detection and attempt to prevent progression to surgical NEC is imperative.

The proposed research aims to qualify the most accurate combination of machine learning methods to ultimately design an automated and computerised way of interpreting the contents of the AR (and hopefully AUS images in future). This will provide the shrinking radiology workforce with the capability to improve efficiency and effectiveness in an environment with increasing patient numbers and mounting complexity of clinical work. The efficiency lies in the dynamic interplay of cross-referencing classifying features through the combination of different networks; e.g. Resnet18, Densenet, and enabling the generation of CAM (also called heatmaps in our case colour maps of specific features). This is achieved through multiple interconnected dense block layers pooling layers in between them and then calculating the average of class-wide features. Within the model will be the required NEC parameters to train and to outperform any other models currently present in the literature. This paper is organized as follows: Section II provides an overview of Diagnosing NEC; Section III presents the clinical imaging features. Section IV presents the research in medical image classification, while Section V shows the proposed model, and finally the conclusions and future work.

\section{Diagnosing NEC}

Necrotizing enterocolitis (NEC) is a potentially lifethreatening condition in neonates. Hence fast and accurate diagnosis of NEC is vital as delays in intervention increases cost [12] and risk factors for poor outcomes; such as surgical NEC, short gut syndrome, and death [3]. Improving accuracy of diagnosis is vital in preventing unnecessary or prolonged treatment with parenteral nutrition and antibiotics, with both being associated with poor outcomes. Diagnostic ambiguity is a problem in many cases of NEC that does not exhibit clinical signs of definitive disease [15]. Hence, accurate diagnosis and ongoing monitoring for NEC has remained highly dependent on imaging to move past suspected disease to medically confirmed NEC [16].

The most commonly used definition case definition for NEC is from the Vermont Oxford Network (VON) [17], see Table 1. The definition is based on the revised Bells Criteria[ and has been adapted to consider spontaneous intestinal perforation (SIP). Various other definitions have been suggested including various subgroups of NEC [5], [15], [21]. Recently the "Two out of three rule" (see Table 1) has been proposed by the International Neonatal Consortium as a new case definition for Preterm NEC [15]. With the INC suggesting the Bell's staging criteria should only be used to stage the severity of NEC and guide treatment decisions; and not as a case definition for NEC [15].

TABLE I. TWO CURRENT DIAGNOSTIC DEFINITIONS FOR NEC

\begin{tabular}{|c|c|}
\hline VON NEC definition[17] & $\begin{array}{l}\text { 'Two out of three' rule for } \\
\text { preterm NEC[5], [15] }\end{array}$ \\
\hline $\begin{array}{l}\text { One symptom of: } \\
\text { 1. Green/yellow (bile stained) gastric } \\
\text { aspirates. } \\
\text { 2. Abdominal distension. } \\
\text { 3. Blood in stools. } \\
\text { Combined with } \\
\text { One if the following radiological } \\
\text { finding of: } \\
\text { - Pneumatosis intestinalis } \\
\text { - Hepato-biliary gas } \\
\text { - Pneumoperitoneum }\end{array}$ & $\begin{array}{l}\text { Step } 1 \text { Differential diagnosis of } \\
\text { NEC by exclusion: } \\
\text { Exclude the following conditions } \\
\text { before applying the inclusion } \\
\text { criteria: } \\
\text { 1. Spontaneous intestinal perforation } \\
\text { or similarly known as focal } \\
\text { intestinal perforation. } \\
\text { 2. Complex congenital heart } \\
\text { diseases (e.g. HLHS). } \\
\text { 3. Infants taking }<80 \text { mL/kg of } \\
\text { enteral feeding without symptoms of } \\
\text { feeding intolerance. } \\
\text { 4. Late preterm and term infants, } 36 \\
\text { weeks' gestation and older. } \\
\text { Step } 2 \text { Inclusion criteria for } \\
\text { preterm NEC: } \\
\text { Patient has a distended abdomen, } \\
\text { ileus and/or bloody stools and meets } \\
\text { two of the following findings: } \\
\text { - } \quad \text { Pneumatosis and/or portal air } \\
\text { by AUS or abdominal X-ray. } \\
\text { - Three days or longer persistent } \\
\text { platelet consumption } \\
\text { ( }<150 \AA \sim 109 / \mathrm{L} \text { ). } \\
\text { 3. Infant's actual age at disease } \\
\text { onset more in keeping with } \\
\text { NEC than SIP ( }>2 \text { weeks). } \\
\text { Ischemia-driven NEC the } \\
\text { estimated onset is within the } \\
\text { first } 30 \text { days of life. }\end{array}$ \\
\hline
\end{tabular}

\section{Clinical Imaging Features}

\section{A. Consensus on NEC and radiology}

In a recent multi-specialist survey, Ahle et al. 2018 [22], 202 clinicians had a $90 \%$ agreement that abdominal radiography (AR) is the first-line imaging modality and it contains the most clinically recognised features signs to identify NEC. There was a resounding agreement on the importance of AR biomarkers in diagnosing, monitoring and guideline decisions on surgery.

\section{B. Features of NEC on abdominal radiography}

Traditionally AR has been the mainstay of imaging and Table 2 illustrates the diagnostic features radiologists look for when reporting on portal venous gas (PVG), pneumatosis intestinalis (PI) or Pneumoperitoneum.

It can demonstrate distended loops of bowel filled with gas, ileus or thickening of the bowel wall. These are common but non-specific signs. The presence of (PVG) or (PI) is considered virtually pathognomonic for NEC. Pneumoperitoneum is challenging to detect on a neonatal film but if seen is indicative of bowel perforation. A horizontal beam cross-table lateral film with the infant placed in the left lateral decubitus position is preferred (when clinical suspicion 
is high for bowel perforation) to the typical anterior-posterior abdominal film. It is essential to highlight that if abdominal ascites is present, it may mask most radiographic findings suggestive of SIP or NEC [16].

TABLE II. NEC RADIOGRAPHICAL DIAGNOSTIC FEATURES

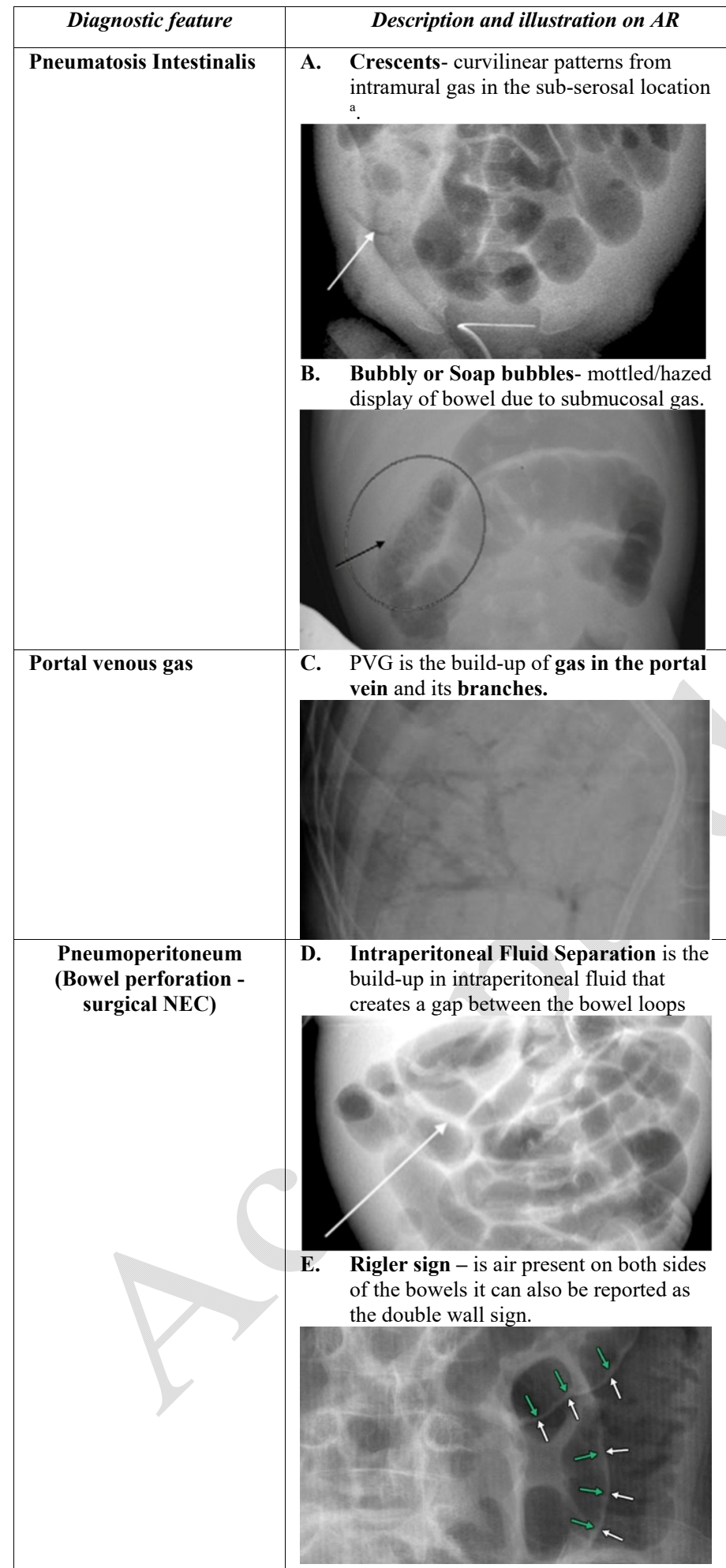

F. Football sign - free-air creating pressure and extension in the peritoneal cavity. The falciform ligament (1) can potentially be outlined.

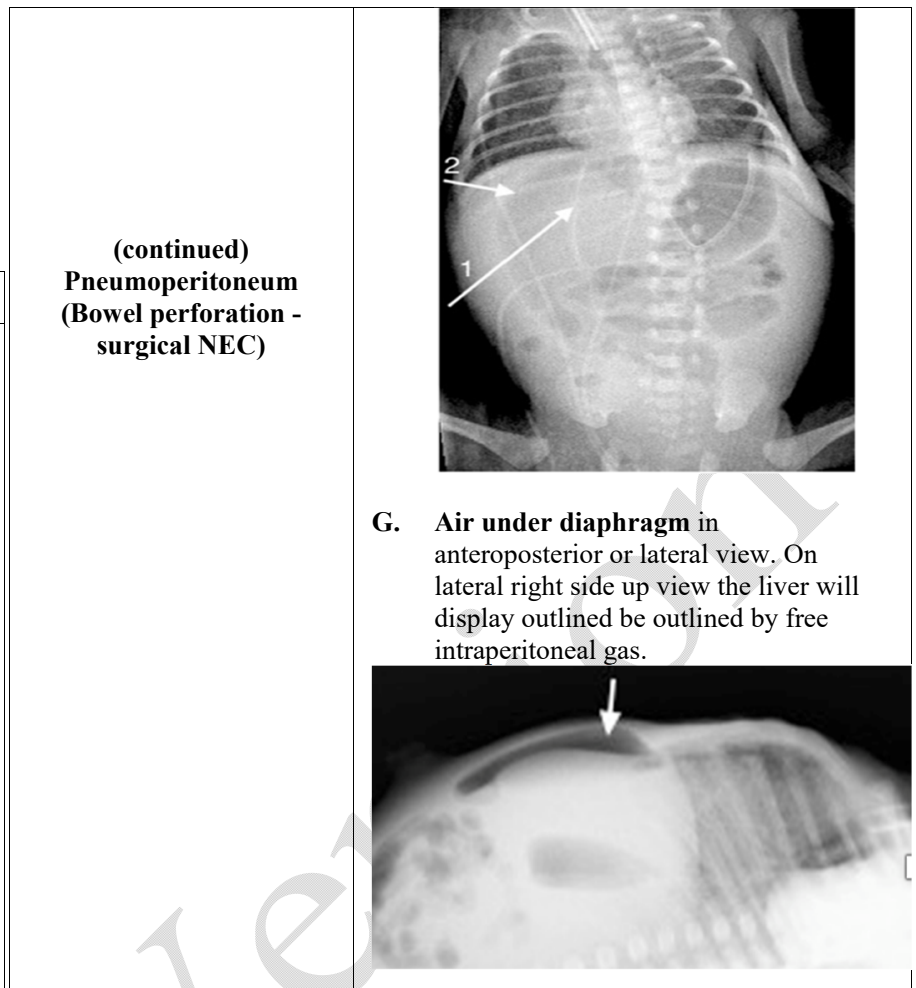

H. Triangle sign: air trapped between bowels and mostly seen above the liver.

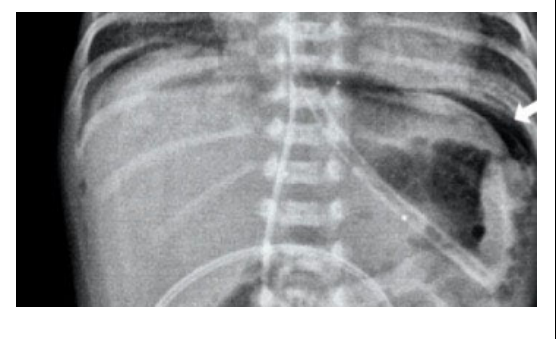

A-H illustration sources.[23], [24]

\section{Need for CAM in neonatal radiology}

The recognized AR visualized pathology in NEC have a good positive predictive value but low sensitivity[25], [26]. Frequently in clinical practice, however, the changes may be subtle and difficult to interpret. Other more nonspecific signs are frequently misjudged as a sign of NEC, and the clinical experience of the treating physician can be vital in safeguarding the correct interpretation and reporting of features. Currently, there is no training in the interpretation of AR signs in NEC offered to clinicians[16]. Imaging reporting remains a challenge, as nonspecific results do not exclude the presence of NEC[27]. Various studies[28], [29], highlights the low agreement and reproducibility in the consistency of reporting of AR signs among clinicians and also among different professional groups. CAD can offer a solution in both training and improving inter-expert agreement in radiological reports. Furthermore, as images automatically get labelled and reviewed by a radiologist (ground truth) as part of clinical processing this can generate big data for real-world evidence studies.

In the recent Clinical Radiology UK Workforce Census Report of 2018[30], it has again highlighted the ongoing rise in the demand for radiology services. Whereas the day-to-day clinical demands is becoming more complex. Unfortunately, the workforce growth is not keeping up with the clinical need, 
a telling sign is the vacant consultant positions that cannot be sourced and the spiralling cost on outsourcing, insourcing and temporary agency staff.[31] This comes at a time where the workforce needs to be nimble to adapt to new technologies, keep abreast with advances and motivate for these innovations to support the NHS. [32]

Daily clinical practice demands that radiologists and clinicians must balance, often competing interests, requests for their review[33]. The cognitive strains of reviewing information across systems to inform treatment decisions repeatedly and rapidly throughout the day are mentally taxing[34]. Hence, it's not surprising when unknowingly various cognitive bias develops. These mental shortcuts are coping mechanisms to enable medical staff to keep up with an ever-increasing workload[35]. But this can work counter productively when cognitive biases develop[33].

Neonatology and paediatrics lack computer-aided detection software (CAD) for neonatal imaging that can help screening, detection and ensuring standardised reporting of imaging findings[36], [37]. Furthermore, novel imaging modalities like AUS remains underutilised due to the lack of expertise in its use and reporting[36], [37]. Highlighting a further area for CAM to assist the integration of new point of care ultrasound (POCUS) to overcome underutilisation[26], [36], [37].

\section{STATE-OF-THE-ART IN AR RELEVANT TO NEC}

In recent years different institutions have released datasets to assist the deployment and development of intelligent technologies in medicine[38]. Deep learning approaches, especially convolutional networks, have quickly established itself as the approach of choice when analyzing medical images[39]. When reviewing the research in X-rays, most of the work respiratory medicine to classify chest radiographs $(\mathrm{CxR})$ due to large synthetic datasets of $(\mathrm{CxR})$ been made available publicly [38].

When considering methods of medical imaging classification, conventional machine learning model should still be considered[39]-[41]. As it can achieve high accuracy of classification as the full extent of the radiological features can be handcrafted and image quality can be optimized in preprocessing to overcome noisy images.

Looking at studies deploying deep learning in chest CxR Cicero et al. 2017, demonstrated that CNN algorithms can attain clinically useful performance even with modest data sets. Using GoogLeNet CNN to identify and eliminate common abnormalities an accuracy of $75 \%$, for consolidation $(\mathrm{n}=214), 78 \%$ for pneumothorax $(\mathrm{n}=167) 82 \%$, for pulmonary edema $(n=356), 80 \%$, cardiomegaly $(n=482)$ and $91 \%$ pleural effusion $(\mathrm{n}=782)$ was achieved [42].

To aid automatic tuberculosis detection, Hwang et al. 2016, created a CAD system centred on deep CNN for automatic tuberculosis identification. The algorithm produced heat maps to highlight regions of disease. The model required large sets of labelled $\mathrm{CxR}$ and utilizing the optimisation through transfer learning to achieve a screening performance of $0.88,0.93$ and 0.96 in three real field datasets [43].

Training a deep CNN from start to finish is challenging as it necessitates big sets of labelled data for training and a high level of knowledge to produce appropriate merging. A substitute is to modify an existing $\mathrm{CNN}$ with pre-training from a large database of labelled AR films. Tajbakhsh et al. 2016, showed that deeply modified CNN's is able to outperform fully trained CNN's and are effective in medical imaging analysis when small amounts of training data is available[40]. Kumar et al. 2016 achieved 80\% accuracy in classification. This was achieved using the Local Binary Pattern (LBP) feature extraction and the Support Vector Machine (SVM) classification algorithms. Demonstrating that an ensemble of modified CNN's achieves better accuracy than fully trained or established CNN's [41].

Newer models include ResNet, Densenet, Inception and VGG to improve the efficiently classify images using convolutional neural networks. Rakshit et al. 2019 showed that by fine-tuning the Resnet model, better performance was achieved with fewer boundaries to train [44]. ResNet[45] was the first model to solve the vanishing gradient problem by reprocessing the activations of the preceding layers ensuring that the layer succeeding learns its weights more accurately. Huang et al. 2019[46], also later resolved the vanishing gradient problem with the Densenet model's interlayer connection architecture. As an alternative to adding a layer, Densenet has a direct link between succeeding layers, hence so all layers have direct contact to the initial input and signal gradients of the loss function.

\section{THE PROPOSED MODEL}

Fig 1, gives an overview of the suggested model to compare the performance of conventional machine learning techniques with deep learning based approach. Inputs can use either AR or static AUS with assigned image-disease-features-labels for training. Outputs classes (various signs of NEC pathology) are generated, which gets assigned a colour to map the pathology as $\mathrm{CAD}$ for the clinician (radiologist or neonatologist) to review the findings. Based on the findings of features the model will utilise various NEC definitions to predict if it meets the diagnostic criteria for medical NEC and if there are suggestive features that it's progressed to surgical NEC.

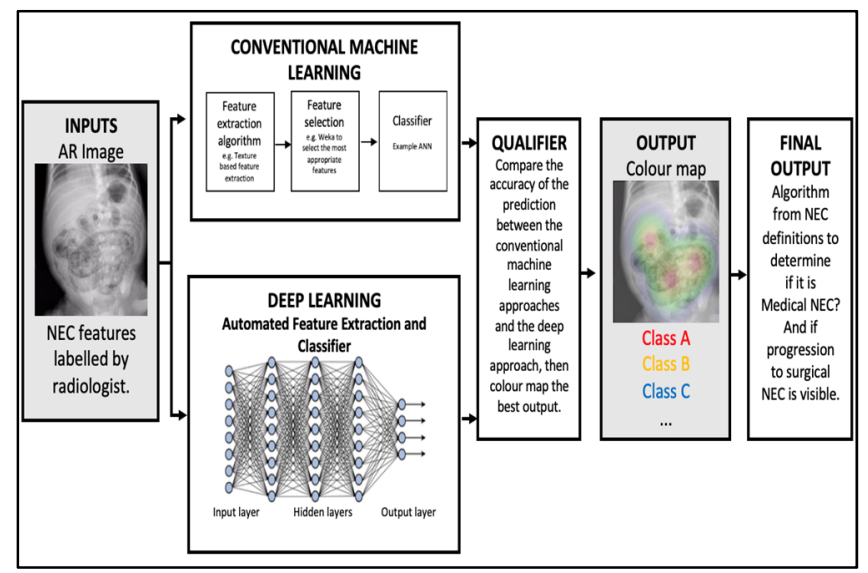

Fig. 1. The full comparative model for NEC imaging CAD and NEC prediction.

Fig 2, illustrates the proposed deep learning based model, using Densenet for example. This model can use either AR or static AUS as an input using image-disease-features-labels to train the model. It generates the required outputs classes, after 
that each class is associated with a suitable colour map and then presented as translucency of colour in the outputs. The architecture of the proposed model has an adaptive DenseNet to generate feature classes and then assigning each class with a specific colour to generate a colour map that represent the classified features.

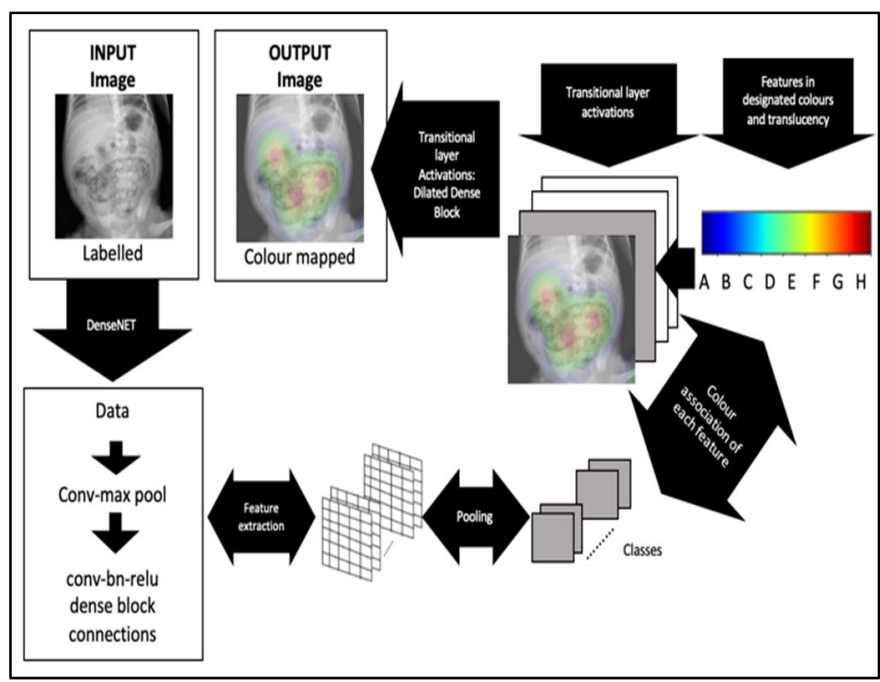

Fig. 2. The Proposed Deep Learning Based Model

\section{CONCLUSION}

As the clinical radiology workforce is shrinking and the workload on radiologists are mounting, it is imperative that emerging technology such as CAD and machine learning be deployed to support the radiology service and reinforce clinical standards. In the case of NEC, it may provide that pivotal step needed to enable the better utilisation of POCUS and generate the real-world evidence (RWE) needed to validate new imaging techniques and diagnostic case definitions.

It is improbable that conventional medical research methods will advance radiology and NEC research as swiftly and cost efficiently as machine learning based technology can. Even though the randomised control trial is still seen as the benchmark for research to determine causation, observational trials have received revived interest as intelligent approaches have shown to be robust for multivariable analysis and manages the issue of confounders. Machine learning based technology can facilitate better mining of data, overcome bias (offering objective processing) to give improved insights. Hence, the future work will focus on testing and validating the proposed model on significant amount of data to provide the right support for the healthcare system and clinicians in detecting NEC.

\section{ACKNOWLEDGMENT}

JvD was awarded an educational grant by NEC UK (http://www. necuk.org.uk) that partially supported her PhD research, for the academic year 2018-2019.

\section{REFERENCES}

[1] C. Battersby, N. Longford, S. Mandalia, K. Costeloe, N. Modi, and UK Neonatal Collaborative Necrotising Enterocolitis (UKNC-NEC) study group, 'Incidence and enteral feed antecedents of severe neonatal necrotising enterocolitis across neonatal networks in England, 2012-
13: a whole-population surveillance study', Lancet Gastroenterol. Hepatol., vol. 2, no. 1, pp. 43-51, 2017.

[2] S. Eaton, 'Necrotizing enterocolitis symposium: Epidemiology and early diagnosis', J. Pediatr. Surg., vol. 52, no. 2, pp. 223-225, Feb. 2017.

[3] J. R. Robinson, C. Kennedy, K. J. van Arendonk, A. Green, C. R. Martin, and M. L. Blakely, 'Neurodevelopmental considerations in surgical necrotizing enterocolitis', Semin. Pediatr. Surg., vol. 27, no. 1, pp. 52-56, Feb. 2018.

[4] N. J. Hall, 'Necrotising enterocolitis: better data, still many questions', Lancet Gastroenterol. Hepatol., vol. 2, no. 1, pp. 6-7, Jan. 2017.

[5] P. V. Gordon, J. R. Swanson, B. C. MacQueen, and R. D. Christensen, 'A critical question for NEC researchers: Can we create a consensus definition of NEC that facilitates research progress?', Semin. Perinatol., vol. 41, no. 1, pp. 7-14, Feb. 2017.

[6] S. M. Gephart and W. M. Eklund, 'NEC Symposium: A transdisciplinary approach to tackle NEC', Adv. Neonatal Care Off. J. Natl. Assoc. Neonatal Nurses, Mar. 2017.

[7] C. Battersby, T. Santhalingam, K. Costeloe, and N. Modi, 'Incidence of neonatal necrotising enterocolitis in high-income countries: a systematic review', Arch. Dis. Child. Fetal Neonatal Ed., vol. 103, no. 2, pp. F182-F189, Mar. 2018.

[8] S. M. Gephart et al., 'Changing the paradigm of defining, detecting, and diagnosing NEC: Perspectives on Bell's stages and biomarkers for NEC', Semin. Pediatr. Surg., vol. 27, no. 1, pp. 3-10, Feb. 2018.

[9] 'Bliss | Bliss baby report 2015 - England'. [Online]. Available: http://www.bliss.org.uk/babyreport. [Accessed: 30-Mar-2018].

[10] First RECAP preterm-related publication is out - a meta-analysis on the association of preterm birth and adult markers of wealth', EFCNI, 2018.

[11] J. A. Bisquera, T. R. Cooper, and C. L. Berseth, 'Impact of necrotizing enterocolitis on length of stay and hospital charges in very low birth weight infants', Pediatrics, vol. 109, no. 3, pp. 423-428, Mar. 2002.

[12] M. E. Mowitz, D. Dukhovny, and J. A. F. Zupancic, 'The cost of necrotizing enterocolitis in premature infants', Semin. Fetal. Neonatal Med., vol. 23, no. 6, pp. 416-419, Dec. 2018.

[13] B. S. R. Allin, A.-M. Long, A. Gupta, K. Lakhoo, and M. Knight, 'Oneyear outcomes following surgery for necrotising enterocolitis: a UKwide cohort study', Arch. Dis. Child. - Fetal Neonatal Ed., p. fetalneonatal-2017-313113, Nov. 2017.

[14] S. H. Shin et al., 'Surgical Necrotizing Enterocolitis versus Spontaneous Intestinal Perforation in White Matter Injury on Brain Magnetic Resonance Imaging', Neonatology, vol. 110, no. 2, pp. 148154, 2016.

[15] S. M. Gephart et al., 'Changing the paradigm of defining, detecting, and diagnosing NEC: Perspectives on Bell's stages and biomarkers for NEC', Semin. Pediatr. Surg., vol. 27, no. 1, pp. 3-10, Feb. 2018.

[16] R. Soni, A. Katana, J. I. Curry, P. D. Humphries, and A. HuertasCeballos, 'How to use abdominal X-rays in preterm infants suspected of developing necrotising enterocolitis', Arch. Dis. Child. - Educ. Pract., p. edpract-2018-315252, Feb. 2019.

[17] Vermont Oxford Network, '2018 Manual of Operations: Part 2 Data Definitions \& Infant Data FormsV', 2018. [Online]. Available: https://public.vtoxford.org/wp-content/uploads/2017/04/ Manual_of_Operations_Part2_v22-1.pdf. [Accessed: 25-Apr-2018].

[18] R. M. Kliegman, W. A. Walker, and R. H. Yolken, 'Necrotizing enterocolitis: research agenda for a disease of unknown etiology and pathogenesis', Pediatr. Res., vol. 34, no. 6, pp. 701-708, Dec. 1993.

[19] M. C. Walsh and R. M. Kliegman, 'Necrotizing Enterocolitis: Treatment Based on Staging Criteria', Pediatr. Clin. North Am., vol. 33, no. 1, pp. 179-201, Feb. 1986.

[20] J. Neu and W. A. Walker, 'Necrotizing Enterocolitis', N. Engl. J. Med., vol. 364, no. 3, pp. 255-264, Jan. 2011.

[21] P. Gordon, R. Christensen, J.-H. Weitkamp, and A. Maheshwari, 'Mapping the New World of Necrotizing Enterocolitis (NEC): Review and Opinion', E-J. Neonatol. Res., vol. 2, no. 4, pp. 145-172, 2012.

[22] M. Ahle, H. G. Ringertz, and E. Rubesova, 'The role of imaging in the management of necrotising enterocolitis: a multispecialist survey and a review of the literature', Eur. Radiol., pp. 1-11, Mar. 2018.

[23] 'LearningRadiology - necrotizing, enterocolitis, nec'. [Online]. Available: http://learningradiology.com/archives2012/COW\% 20514NEC/neccorrect.html. [Accessed: 24-Jun-2019]. 
[24] Y. T. Yildiz et al., 'Imaging findings in necrotizing enterocolitis', 06Mar-2014. [Online]. Available: https://posterng.netkey.at/esr/viewing/ index.php? module $=$ viewing_poster $\&$ task $=\& \mathrm{pi}=121515$. [Accessed: 24-Jun-2019].

[25] K. Muchantef, M. Epelman, K. Darge, H. Kirpalani, P. Laje, and S. A. Anupindi, 'Sonographic and radiographic imaging features of the neonate with necrotizing enterocolitis: correlating findings with outcomes', Pediatr. Radiol., vol. 43, no. 11, pp. 1444-1452, Nov. 2013.

[26] S. Chen, Y. Hu, Q. Liu, X. Li, H. Wang, and K. Wang, 'Comparison of abdominal radiographs and sonography in prognostic prediction of infants with necrotizing enterocolitis', Pediatr. Surg. Int., vol. 34, no. 5, pp. 535-541, May 2018.

[27] D. Hackam and M. Caplan, 'Necrotizing enterocolitis: Pathophysiology from a historical context', Semin. Pediatr. Surg., vol. 27, no. 1, pp. 11-18, Feb. 2018

[28] K. Markiet, A. Szymanska-Dubowik, I. Janczewska, I. DomazalskaPopadiuk, A. Zawadzka-Kepczynska, and A. Bianek-Bodzak, 'Agreement and reproducibility of radiological signs in NEC using The Duke Abdominal Assessment Scale (DAAS)', Pediatr. Surg. Int., vol. 33, no. 3, pp. 335-340, Mar. 2017.

[29] S. El-Kady, D. Petel, and R. Baird, 'Inter-rater agreement in the evaluation of abdominal radiographs for necrotizing enterocolitis', J. Pediatr. Surg., vol. 49, no. 5, pp. 733-735, May 2014.

[30] 'RCR radiology workforce census | The Royal College of Radiologists'. [Online]. Available: https://www.rcr.ac.uk/clinicalradiology/service-delivery/rcr-radiology-workforce-census. [Accessed: 17-Mar-2019]

[31] 'Clinical radiology UK workforce census 2017 report', Clin. Radiol., p. $40,2017$.

[32] 'Clinical radiology UK workforce census report 2018 | The Royal College of Radiologists'. [Online]. Available: https://www.rcr.ac.uk/publication/clinical-radiology-uk-workforcecensus-report-2018. [Accessed: 20-Jun-2019].

[33] L. P. Busby, J. L. Courtier, and C. M. Glastonbury, 'Bias in Radiology: The How and Why of Misses and RadioGraphics, vol. 38, no. 1, pp. 236-247, Dec. 2017.

[34] A. Murphy, 'Cognitive bias in diagnostic radiology | Radiology Reference Article | Radiopaedia.org', Radiopaedia. [Online] Available: https://radiopaedia.org/articles/cognitive-bias-in-diagnostic -radiology?lang=gb. [Accessed: 24-Jun-2019].

[35] T. Campbell, L. V. Boven, N. Schwarz, and P. Ubel, Too Much Experience: A Desensitization Bias in Emotional Perspective Taking. Vol. 106, No. 272-285, 2014.
[36] J. van Druten, M. Khashu, S. S. Chan, S. Sharif, and H. Abdalla, 'Abdominal ultrasound should become part of standard care for early diagnosis and management of necrotising enterocolitis: a narrative review', Arch. Dis. Child. - Fetal Neonatal Ed., p. fetalneonatal-2018316263, May 2019.

[37] J. van Druten, M. S. Sharif, M. Khashu, and H. Abdalla, 'A Proposed Machine Learning Based Collective Disease Model to Enable Predictive Diagnostics in Necrotising Enterocolitis', in International Conference on Computing, Electronics Communications Engineering (iCCECE), pp. 101-106, 2018.

[38] G. Litjens et al., 'A survey on deep learning in medical image analysis', Med. Image Anal., vol. 42, pp. 60-88, Dec. 2017.

[39] S. M. Anwar, M. Majid, A. Qayyum, M. Awais, M. Alnowami, and M. K. Khan, 'Medical Image Analysis using Convolutional Neural Networks: A Review', J. Med. Syst., vol. 42, no. 11, p. 226, Oct. 2018.

[40] N. Tajbakhsh et al., 'Convolutional Neural Networks for Medical Image Analysis: Full Training or Fine Tuning?', IEEE Trans. Med. Imaging, vol. 35, no. 5, pp. 1299-1312, 2016.

[41] A. Kumar, J. Kim, D. Lyndon, M. Fulham, and D. Feng, 'An Ensemble of Fine-Tuned Convolutional Neural Networks for Medical Image Classification', IEEE J. Biomed. Health Inform., vol. 21, no. 1, pp. 3140, Jan. 2017.

[42] M. Cicero et al., 'Training and Validating a Deep Convolutional Neural Network for Computer-Aided Detection and Classification of Abnormalities on Frontal Chest Radiographs', Invest. Radiol., vol. 52, no. 5, pp. 281-287, May 2017.

[43] S. Hwang, H.-E. Kim, J. J. M.d, and H.-J. Kim, 'A novel approach for tuberculosis screening based on deep convolutional neural networks', in Medical Imaging 2016: Computer-Aided Diagnosis, vol. 9785, p. 97852W, 2016.

[44] S. Rakshit, I. Saha, M. Wlasnowolski, U. Maulik, and D. Plewczynski, 'Deep Learning for Detection and Localization of Thoracic Diseases Using Chest X-Ray Imagery', in Artificial Intelligence and Soft Computing, pp. 271-282, 2019.

[45] K. He, X. Zhang, S. Ren, and J. Sun, 'Deep Residual Learning for Image Recognition', 2016 IEEE Conf. Comput. Vis. Pattern Recognit. CVPR, pp. 770-778, 2016.

[46] Q. Huang, F. Zhang, and X. Li, 'Machine Learning in Ultrasound Computer-Aided Diagnostic Systems: A Survey', BioMed Res. Int., vol. 2018, Mar. 2018. 